\title{
Fourth ventricle stent placement for treatment of recurrent syringomyelia in patients with type I Chiari malformations
}

\author{
Coleman P. Riordan, BSc, and R. Michael Scott, MD \\ Department of Neurosurgery, Boston Children's Hospital, Boston, Massachusetts
}

OBJECTIVE In patients with syringomyelia and type I Chiari malformation (CM-I) who have required reoperation because of persistent, recurrent, or expanding syrinx, the senior author placed a stent from the fourth ventricle to the cervical subarachnoid space in hopes of promoting circulation of CSF out of the ventricle and away from the central canal of the spinal cord. This study was undertaken to determine the long-term success of this operative stratagem in eliminating the syrinx, as well as to document the complications that occurred following stent placement. The technique utilized for placement of fourth ventricle stents is presented.

METHODS The surgical database of the senior author was reviewed to identify all patients who underwent stent placement at a reexploration of a suboccipital decompression for a $\mathrm{CM}$-I conducted for a recurrent or ineffectively treated syringomyelia. The clinical and radiological data of these patients were analyzed to determine long-term efficacy and complications of the procedure.

RESULTS Fourteen patients (average age 10.7 \pm 5.2 years, range 2.6-20.1 years) were identified who met these inclusion criteria. They each presented with recurrent, residual, or expanding syringomyelia following a prior decompression for a CM-I. The reoperation with stent placement was complicated by late stent dislodgement and recurrence or persistence of the syrinx in 2 patients (14\%) and by neurological deficit in 1 patient $(7 \%)$. There was 1 perioperative CSF leak (7\%). In 1 other patient $(7 \%)$, the stent dislodged after surgery but required no further intervention, as the syrinx remained collapsed. Two patients (14\%) required late reoperation for stent replacement when syrinxes recurred. At the most recent imaging follow-up, the stent was positioned appropriately in 12 patients (86\%; average follow-up 6.9 years, range $0.5-18.1$ years), and the recurrent or residual syrinx was eliminated or reduced in size by $75 \%$ or greater in 13 patients (93\%).

CONCLUSIONS The placement of a stent from the fourth ventricle to the cervical subarachnoid space was a highly effective treatment for patients with recurrent, residual, or expanding syringomyelia following an initial decompression of an associated CM-I. The sole neurological complication in this series was related to lysis of arachnoid scar rather than stent placement itself, but inability to maintain fixation of the stent in situ led to further surgery to replace the stent in 2 patients.

https://thejns.org/doi/abs/10.3171/2018.7.PEDS18312

KEYWORDS type I Chiari malformation; syringomyelia; cervical to subarachnoid stent; outcome

$\mathrm{I}$ $\mathrm{N}$ patients with syringomyelia and type I Chiari malformation $(\mathrm{CM}-\mathrm{I})$ who have required reoperation because of persistent, recurrent, or enlarging syrinx, the senior author (R.M.S.) has placed a stent from the fourth ventricle to the cervical subarachnoid space if the second operative exploration demonstrated evidence of obstructed circulation of CSF from the foramen of Magendie. ${ }^{3}$ This study was undertaken to determine the long-term success of this operative stratagem in eliminating the syrinx, as well as to document the complications that occurred following stent placement. The senior author's technique for placement of a fourth ventricle stent is described.

\section{Methods}

This study was approved by the IRB of Boston Chil-

ABBREVIATIONS BCH = Boston Children's Hospital; $\mathrm{CM}=$ Chiari malformation; $\mathrm{CM}-\mathrm{I}$ = type I $\mathrm{CM}$; VP = ventriculoperitoneal. SUBMITTED May 23, 2018. ACCEPTED July 26, 2018.

INCLUDE WHEN CITING Published online October 19, 2018; DOI: 10.3171/2018.7.PEDS18312. 
dren's Hospital $(\mathrm{BCH})$. The senior author's surgical database was reviewed to identify patients who underwent stent placement as part of a reoperation for the treatment of a persistent, recurrent, or enlarging syringomyelia after an initial decompression of an associated CM-I. The hospital records of these patients were reviewed to determine demographic data, presenting symptoms, prehospital treatment, operative information, and late clinical course. Radiographic studies were reviewed to confirm diagnosis and to determine late radiographic outcome.

\section{Results}

The database search yielded 14 patients who underwent placement of a fourth ventricle stent at a second operation for a CM-I associated with a recurrent, persistent, or enlarging syringomyelia. These operations took place over a 20-year period from 1994 to 2014. There were 221 total CM-I decompression procedures performed by the senior author during this period in 196 patients, including 183 primary decompressions $(82.8 \%)$ and 38 reexplorations (17.2\%). The primary procedure included bone decompression, durotomy, and dural graft alone in 136 patients $(136 / 183,74.3 \%)$, and stent placement for associated syringomyelia when the foramen of Magendie was noted to be occluded by a scar or arachnoid web in 47 patients (47/183, $25.7 \%$ ). Among the 38 reexplorations, a stent was placed in 25 procedures in 24 patients $(25 / 38,65.8 \%$; 1 patient with 2 stent placements).

Ten of these patients were excluded from this study
(Fig. 1). Five of the patients with stent placement at reoperation had undergone stent placement during their primary procedure, and were thus not included in this study, as it focused on stents placed during reexploration. Two patients were excluded due to lack of clinical and radiographic follow-up. Three patients were excluded from analysis because of their complexity and confounding comorbidities. Of these, 1 patient was excluded because of a history of chemotherapy and radiation for a nasopharyngeal rhabdomyosarcoma, who suffered a massive intracranial bleed secondary to treatment effects and intracranial aneurysm; the syrinx appeared decompressed at MRI performed at the time of the hemorrhage, but the studies were inadequate to determine ultimate efficacy of the stent. The other 2 patients had complex cranial bone syndromes and concomitant ventriculoperitoneal (VP) shunts that became infected, requiring removal of all retained foreign bodies, including the stents, which themselves showed no evidence of infection. The remaining cohort of 14 patients comprised the study population (Table 1).

\section{Primary Procedure}

The primary procedure was classified as the first attempted CM-I decompression surgery, which in all cases included $\mathrm{Cl}$ laminectomy, suboccipital craniectomy, and dural graft. At the time of initial presentation, patients presented with a number of symptoms and signs: 7 patients (50\%) presented with headaches, $5(36 \%)$ presented with scoliosis, 3 (21\%) with respiratory difficulty, 2 (14\%) with

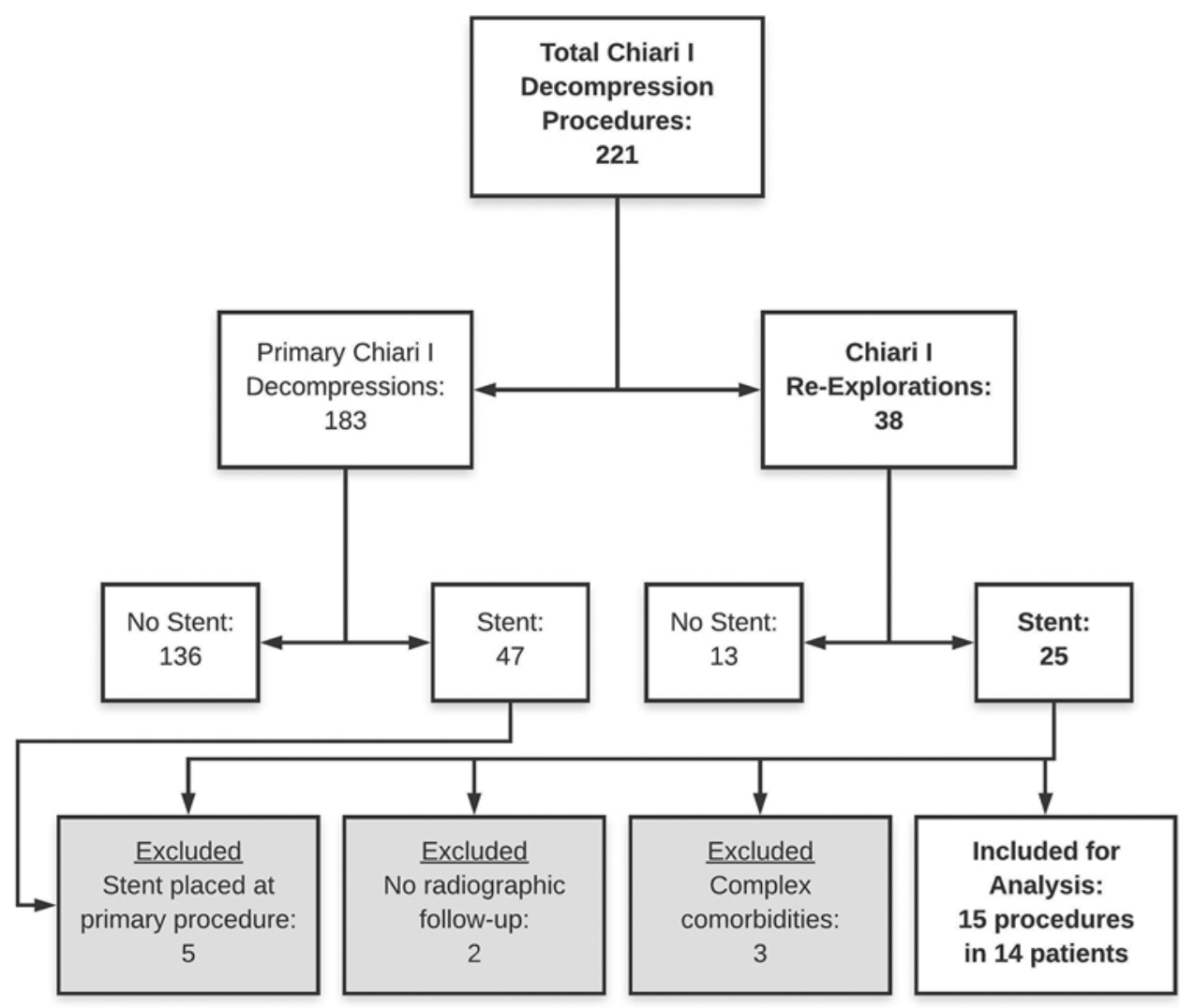

FIG. 1. Patient exclusion flowchart. 
TABLE 1. Clinical courses of the study cohort

\begin{tabular}{|c|c|c|c|c|c|c|}
\hline Case No. & Sex & $\begin{array}{c}\text { Age at Stent Placement } \\
\text { Op (yrs) }\end{array}$ & $\begin{array}{l}\text { Arachnoid Web/Scar } \\
\text { Over 4th Ventricle? }\end{array}$ & $\begin{array}{l}\text { 2nd Reop } \\
\text { Required? }\end{array}$ & $\begin{array}{c}\geq 75 \% \text { Syrinx } \\
\text { Decompression }\end{array}$ & $\begin{array}{l}\text { Total No. of } \\
\text { Procedures }\end{array}$ \\
\hline 1 & $\mathrm{~F}$ & 12.0 & Yes & - & Yes & 2 \\
\hline 2 & $M$ & 4.2 & - & Yes & Yes & 4 \\
\hline 3 & M & 6.9 & Yes & - & Yes & 2 \\
\hline 4 & $\mathrm{~F}$ & 13.7 & Yes & - & Yes & 2 \\
\hline 5 & $M$ & 7.5 & Yes & - & Yes & 2 \\
\hline 6 & $M$ & 14.9 & Yes & - & Yes & 2 \\
\hline 7 & $\mathrm{~F}$ & 20.1 & Yes & - & Yes & 2 \\
\hline 8 & $\mathrm{~F}$ & 12.3 & Yes & - & Yes & 2 \\
\hline 9 & M & 2.6 & Yes & - & No & 2 \\
\hline 10 & $\mathrm{~F}$ & 5.9 & Yes & - & Yes & 2 \\
\hline 11 & M & 6.0 & - & - & Yes & 2 \\
\hline 12 & $\mathrm{~F}$ & 13.8 & Yes & Yes & Yes & 3 \\
\hline 13 & M & 12.9 & - & - & Yes & 2 \\
\hline 14 & $\mathrm{~F}$ & 17.0 & - & - & Yes & 2 \\
\hline Total (\%) & & Mean $10.7 \pm 5.2$ & 10/14 (71\%) & $2 / 14(14 \%)$ & $13 / 14(93 \%)$ & Mean $2.2 \pm 0.6$ \\
\hline
\end{tabular}

hydrocephalus, and 4 (29\%) with other neurological symptoms, which included sensory deficits, paresthesias, and balance disturbance. All initial scans in the 14 patients demonstrated CM-I with associated syringomyelia.

The average age $( \pm \mathrm{SD})$ at initial surgery was $8.7 \pm 5.3$ years, and ranged from 14 months to 19.7 years. There were 7 boys and 7 girls. The primary procedure was performed by other neurosurgeons in 6 patients (43\%), and by the senior author in 8 patients $(57 \%)$. The primary procedures were complicated by postoperative CSF leak, overt wound infection, or pseudomeningocele in 3 patients (21\%). Of these, 2 had been initially operated on at outside institutions. None of the patients underwent additional CM-I surgery prior to stent placement.

\section{Secondary Procedure With Stent Placement \\ Presentation at Reoperation}

The clinical histories and pre-reoperative and late postreoperative MRI studies of 5 representative patients are included in Figs. 2-6. All 14 patients underwent reexploration of the original suboccipital craniectomy and $\mathrm{Cl}$ laminectomy and placement of a stent from the fourth ventricle to the cervical subarachnoid space over a 20 -year period from 1994 to 2014. The operative technique is described below. Ten $(71 \%)$ of these operations also included partial C2 laminectomy. The average patient age at the time of reoperation was $10.7 \pm 5.2$ years (range 2.6-20.1 years).

The average time interval between primary and secondary surgery was $2.0 \pm 1.7$ years (range $0.4-5.1$ years). All 14 patients presented with persistence, recurrence, or expansion of the initially diagnosed syrinx. Additionally, $7(50 \%)$ had new-onset headaches, $5(36 \%)$ exhibited progression of scoliosis, and $2(14 \%)$ had new sensory changes in their extremities. No patients, including the 2 patients initially presenting with hydrocephalus, required VP shunt placement.

\section{Operative Technique}

A standardized technique for stent placement was used in all cases. The region of the fourth ventricular outflow (foramen of Magendie) was identified after lysis of any arachnoid adhesions in the adjacent subarachnoid space. In $10(71 \%)$ of these reoperations, the foramen of Magendie was scarred shut by dense adhesions at the foramen or in the surrounding arachnoid, and great care had to be taken when the arachnoid scar was opened in order to not damage the posterior inferior cerebellar artery or the subjacent
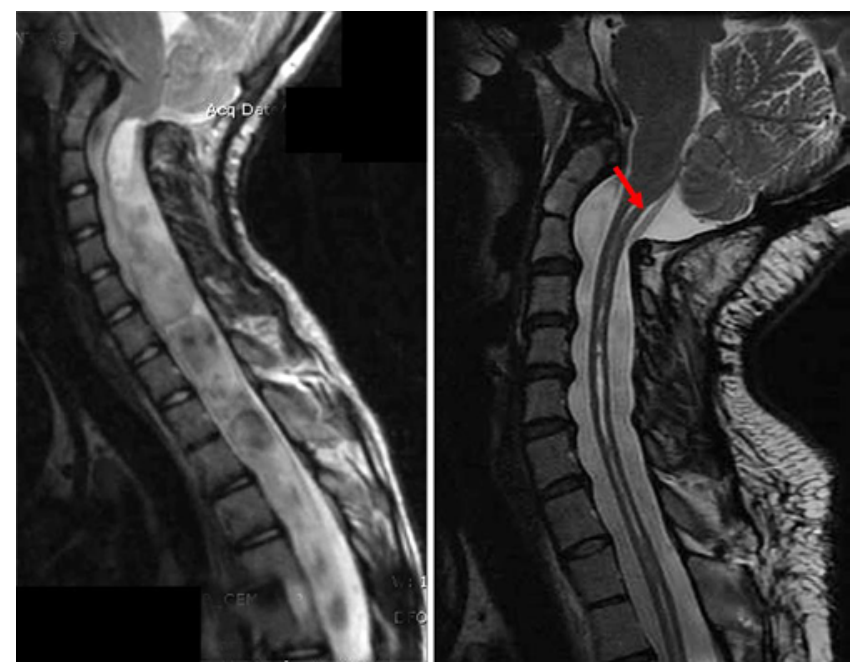

FIG. 2. Case 4. This 12-year-old girl initially presented with scoliosis associated with a large syrinx and CM-I. Six months later her scoliosis had worsened and MRI revealed an enlarging, holocord syrinx (left). At reoperation, a veil of arachnoid was found at the foramen of Magendie and a stent was placed from the fourth ventricle to the cervical subarachnoid space. Follow-up MRI 13.6 years after stent placement (right) showed the stent in position (arrow) and stable decompression of the syrinx. Figure is available in color online only. 


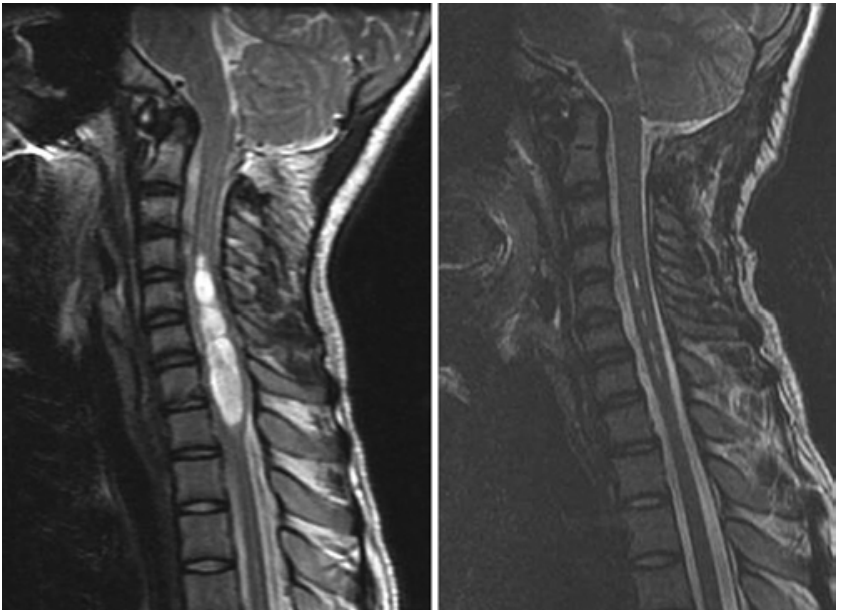

FIG. 3. Case 6. This 9-year-old boy presented with ventriculomegaly and a large syrinx. Following CM-I decompression, his ventricular system and syrinx became reduced in size, but MRI 5 years later (left) revealed syrinx recurrence. At reoperation, the foramen of Magendie was noted to be scarred shut. The scar was lysed and a stent was placed. MRI 6 months after reoperation (right) showed almost complete decompression of the syrinx. His neurological examination 3.5 years after reoperation was normal, but no further follow-up imaging was obtained and the patient was lost to follow-up.

brainstem. In 2 patients (14\%), a veil of arachnoid occluded the otherwise normal-appearing foramen, in both cases a finding missed at the original operation; in 3 patients (21\%), despite careful inspection of the fourth ventricle outflow, no occlusive process could be identified at reoperation. In these 3 cases, it was assumed that there was probable "functional" obstruction at the fourth ventricle outlet either related to scarring that had been lysed as part of the exposure and exploration, or related to intermittent pulsatile occlusion of the outlet by the cerebellar tonsils. Because the first "standard" decompression was known to be unsuccessful, it was believed that enhanced outflow of CSF from the fourth ventricle by means of stent placement would aid in decompressing the syrinx. A Pudenz ventricular catheter (Integra NeuroSciences) was used for the stent because of its slightly narrower external diameter $(2.5 \mathrm{~mm})$ compared to a standard ventricular catheter, its translucent walls enabling confirmation of CSF flow within the catheter after placement, and the centimeter markings on its external surface ensuring that the catheter was cut and placed at appropriate length within and without the fourth ventricle. A catheter length of 4-5 cm was typically utilized, with approximately $3 \mathrm{~cm}$ placed within the fourth ventricle along its floor, and the distal end placed under carefully preserved arachnoid over the dorsal upper cervical spinal cord. If the subarachnoid space dorsal to the upper cervical cord was occluded by scar, a small amount of the upper border of the C2 lamina was removed and the dura exposure lengthened inferiorly to visualize clear subarachnoid space. The catheter was then secured in place using two 6-0 silk sutures taken through each side of the catheter wall at the level of the foramen of Magendie and through focally coagulated pia of the adjacent medial cerebellum. The dura was then closed with an AlloDerm (LifeCell Corp.) patch graft.
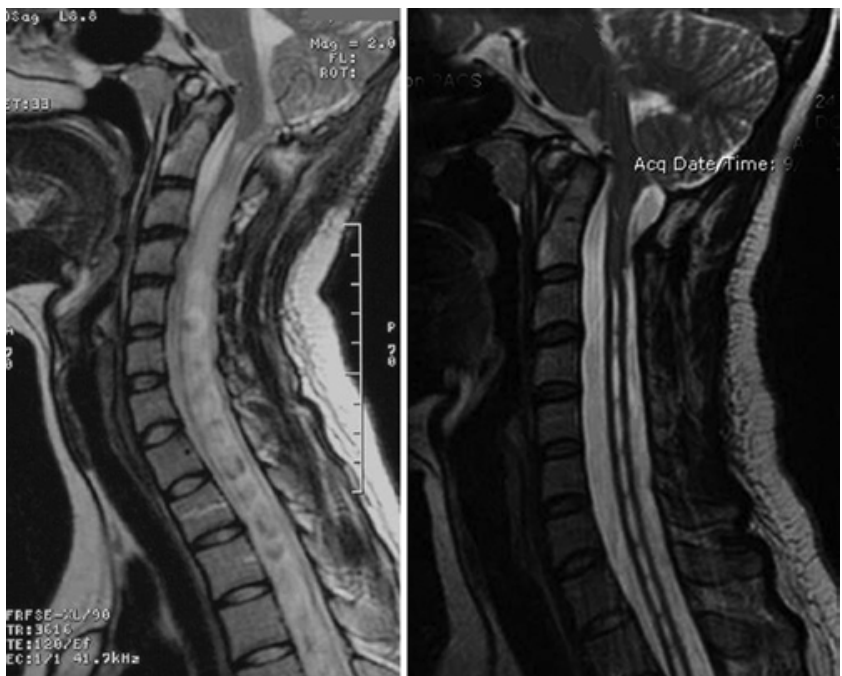

FIG. 4. Case 7. This 14-year-old girl was operated on at an outside hospital for CM-I and minimal dilation of the central canal. Six years later, she presented there again with a large syrinx. Reoperation was complicated by wound abscess and meningitis, and postoperative MRI revealed a persisting and enlarging syrinx (left). At her third operation 3 months later at $\mathrm{BCH}$, an extensive scar in the dorsal subarachnoid space and at the foramen of Magendie was encountered and lysed, and a stent was placed. Postoperatively, the patient suffered a unilateral partial dorsal column deficit believed to be from superficial devascularization when the dense scar was lysed. MRI 4.8 years after stent placement (right) revealed almost complete decompression of her syrinx and the stent in good position. A mild, right, upper extremity dorsal column and lateral spinothalamic tract deficit persisted at last clinical follow-up 3.5 years postoperatively.

\section{Operative Complications}

There were 4 (28.6\%) postoperative complications related to stent placement surgery. Two patients experienced stent dislodgement with return or persistence of their syringomyelia. In the first patient, the stent never successfully decompressed the syrinx even when in place, signifying a failure of the stent procedure (7.1\%). The second patient developed cervical nerve root irritation at the locus of the dislodged stent in addition to a second recurrence of the syringomyelia. A third patient initially operated on elsewhere and whose first procedure was complicated by a serious wound infection and meningitis had a mild unilateral dorsal column deficit after stent placement surgery, almost certainly related to vascular injury to the dorsal cervical spinal cord during the lysis of extensive adhesions in the arachnoid prior to stent placement (Fig. 4). We believe that this deficit was unrelated to stent placement per se, because the scarring occluding the subarachnoid space and foramen of Magendie secondary to the patient's severe initial postoperative infection would have required lysis at a reoperation regardless of an attempt to place a stent. One patient $(7 \%)$ had a CSF leak requiring placement of a lumbar drain that treated the leak successfully without further sequelae.

\section{Clinical Follow-Up}

Average clinical follow-up, available in all 14 patients, was $8.0 \pm 5.5$ years (range 2.0-19.1 years) following reop- 


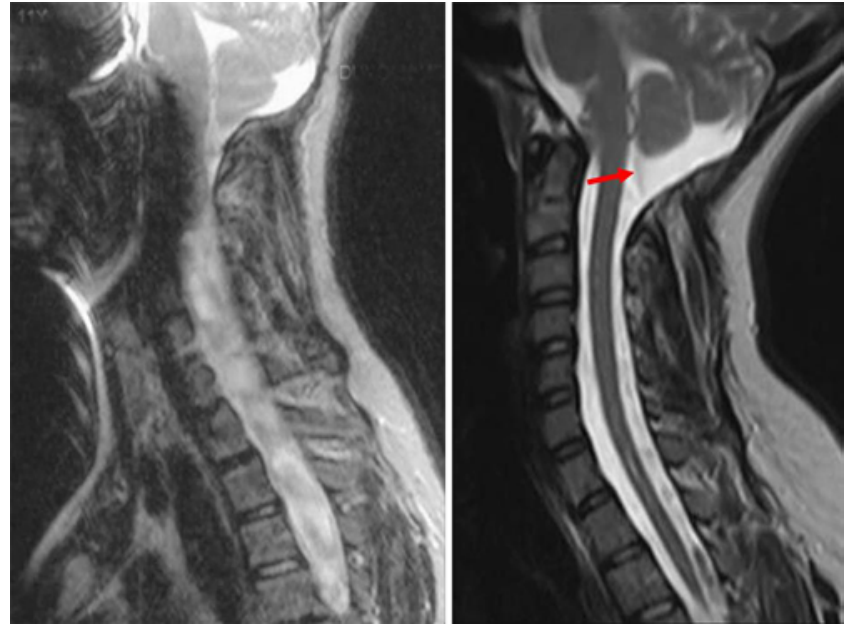

FIG. 5. Case 8. This 11-year-old girl initially presented with scoliosis, a CM-I, and syringomyelia. One year after surgery at an outside institution, MRI (left) demonstrated an enlarged holocord syrinx. At reoperation at $\mathrm{BCH}$, dense arachnoid bands and an occlusive web over the foramen of Magendie were found; the adhesions and webs were lysed, and a stent was placed. MRI 6.1 years following stent placement (right) demonstrated complete collapse of the syrinx and a stent (arrow) in stable position. Figure is available in color online only.

eration for stent placement. Of the 14 reoperated patients, $12(86 \%)$ required no further procedures. Two patients (14\%) required further surgery: 1 patient described above underwent an additional operation to reposition her dislodged stent after she developed both syrinx recurrence and cervical nerve root irritation. The dislodged stent was easily removed and replaced in a subsequent operation, from which the patient has done extremely well after a 3 -year follow-up. One patient with a complex Apert/Pfeiffer syndrome variant underwent a total of 4 procedures: an initial CM-I decompression performed by another surgeon, a procedure to place a stent using a Spetzler lumbar
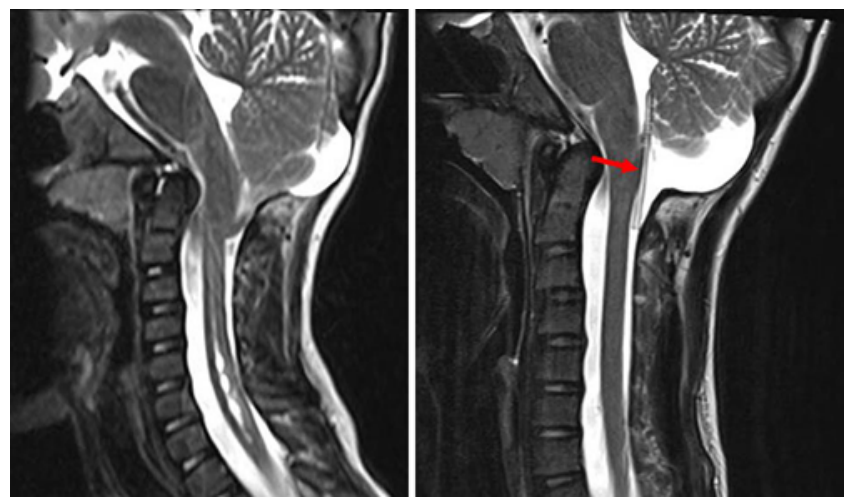

FIG. 6. Case 10. This 3.5-year-old girl presented with severe brief headaches and MRI demonstrating a CM-I with tonsils at the C2-3 level. At her initial surgery at $\mathrm{BCH}$, the tonsils were coagulated and shriveled as part of the decompression. At age 6, the headaches recurred and MRI (left) showed a new syrinx with apparent outflow obstruction of the distal fourth ventricle. At reoperation at $\mathrm{BCH}$, a web of arachnoid occluded the distal fourth ventricle and a stent was placed. MRI 5.5 years after stent placement (right) demonstrated collapse of the syrinx and the stent (arrow) in good position. Figure is available in color online only. peritoneal catheter (Integra NeuroSciences) to treat a persisting syrinx, a procedure to replace the Spetzler catheter because of a persisting syrinx that had to be aborted because of acute airway obstruction, and a final successful stent replacement with a Pudenz catheter. Follow-up MRI 9 years later demonstrated complete resolution of the syringomyelia. ${ }^{1,4}$ In this case, the Spetzler catheter was believed to have too small a lumen diameter to allow adequate outflow from the fourth ventricle, but replacement with the larger-diameter Pudenz catheter led to excellent results.

The patient who experienced the unilateral dorsal column deficit made a good recovery following physical therapy, with only a mild persisting right-sided sensory deficit.

In 1 additional patient, the stent could not be visualized on routine follow-up imaging 1 year postoperatively. Despite stent migration, the syrinx remained completely collapsed and the patient reported no symptoms. We postulate that the stent was in place long enough to decompress the syrinx and establish a tract creating flow out of the fourth ventricle, and no further intervention was required. Therefore, 3 patients (21.4\%) ultimately experienced stent dislodgement, although only 1 required additional surgery to reposition the stent.

Five patients $(35.7 \%)$ have reported occasional neck pain and/or headache following their procedures, none requiring further operative interventions. All patients were leading independent lives at the time of follow-up.

\section{Radiographic Findings}

Average imaging follow-up was $6.9 \pm 5.3$ years (range $0.5-18.1$ years). All patients had available pre- and postoperative MR images. At the time of last postoperative MRI, the stent was clearly visible and in position for 12 patients (86\%), but in 2 patients the stent could not be clearly visualized and was likely dislodged. Only one of these patients required reoperation with new stent placement (noted above), while the other showed stable collapse of his syrinx in the absence of a visible stent. Last available imaging demonstrated virtually complete absence of the syrinx in 13 patients (93\%). In 1 patient with a short, focal syrinx that was initially $16.5 \mathrm{~mm}$ in length, the syrinx was slightly longer $(19 \mathrm{~mm})$ at follow-up, but reduced in width (8.6 vs $9.1 \mathrm{~mm})$.

\section{Discussion}

Persistent, recurrent, or enlarging syringomyelia is an important indication for reoperation in patients who have undergone prior surgery for an associated CM. We have previously published our experience with reoperations for patients with CMs, noting that young patients and syndromic patients, typically those with craniosynostosis syndromes, are more likely to require second operations for the CM. ${ }^{3}$ In cases of recurrent, persisting, or expanding syringomyelia, we have felt that inadequate outflow of CSF from the fourth ventricle was playing a major role in the development of this postoperative complication, and that merely repeating the bone and dural decompression would not lead to successful decompression of the recurrent syrinx. The need to consider fourth ventricle stent 
placement in this situation was noted previously by Venes and VanGilder, and we have found stent placement to be an effective technique to treat a recurrent syrinx when scarring or partial occlusion of the foramen of Magendie is likely to be a significant factor in syrinx recurrence. ${ }^{2,5}$

The decision by the senior author to place a stent at reexploration was based on the reported findings at the primary procedure; knowledge of any complications following the first operation, such as infections or bleeding (which might have led to scarring in the subarachnoid space); and most importantly, the finding at reoperation that the fourth ventricular outflow was occluded by scar, which was believed by the senior author to likely recur if simply lysed. As noted above, however, stents were placed in 3 patients when it appeared that no explanation other than partially impeded CSF flow at the foramen of Magendie had led to failure of the primary procedure. Placement of a fourth ventricle stent was ultimately effective in treating recurrent or residual syringomyelia in 13 patients (93\%), although in 2 patients the stent had to be repositioned, one because of postoperative migration and syrinx re-recurrence and the other because the catheter used initially proved to be too narrow for adequate flow.

During the study period, two reoperations were performed for recurrent/residual syrinx in which no stent was placed. In one of these, a stent was not used because it was clear that the initial dural and bone decompression had been inadequate, and in 1 patient the ventricular outflow and much of the fourth ventricle itself were wide open at the reexploration.

In one of the senior author's own primary cases that required reoperation and stent placement, the cerebellar tonsils were adherent to one another at reexploration, blocking CSF outflow from the foramen of Magendie, suggesting that an over-vigorous pial coagulation of the medial descended tonsils contributed to this obliterative scarring. Since this phenomenon was noted, subsequent tonsillar coagulation has been limited to the outer, inferior, and dorsal portions of the herniated tonsil rather than to its medial aspect adjacent to the foramen of Magendie. Scrupulous hemostasis at the original operation and measures to reduce operative infection risk, including the prompt management of pseudomeningoceles and CSF leak, will also likely reduce the need for reoperation and stent placement.

For several decades, the senior author routinely placed stents for any patient with syringomyelia, but it became apparent that this technique was not necessary in many primary cases unless a web or scarred arachnoid blocked the foramen. As proof of principle, 2 patients who were not included in this study because a stent had been placed at the primary procedure developed recurrent syringomyelia when their primarily positioned stent became dislodged; following a reoperation for replacement of the dislodged stent, the syrinxes again decompressed. Although syrinxes were treated quite successfully by this technique, it was difficult to prove that the stent played any role in the initial syrinx decompression, given that an adequate decompression of the CM will often result in the same outcome. It is also apparent from this case review that the technique described above for securing the stent in place is not foolproof. Three of these stents were noted to be- come dislodged during follow-up. In one of these patients the catheter could not be visualized on late MRI with the syrinx remaining decompressed, and the authors have assumed that the catheter was in place long enough for a permanent CSF conduit to have been established out of the fourth ventricle along the catheter's former tract. When the stent dislodged in the other 2 patients, however, the syrinx recurred or persisted. In 1 patient, the previously decompressed syrinx returned, which was successfully treated by another operation to replace the stent. In the other patient, the stent had been unsuccessful in decompressing the small, focal syrinx even when in place at 1 month after the operation. As a result, it was not felt that an additional procedure to replace the stent was indicated, and the syrinx has remained stable upon 5-year follow-up. This case is considered to be the one true failure of the stenting technique, as the syrinx was not decompressed while the stent was patent. The senior author has been reluctant to secure these stent catheters to the dura, on the theory that with neck movement the catheter, in turn, would slide up and down within the fourth ventricle, possibly injuring its floor. A more effective technique to secure the stent in place, however, would be a welcome addition to the operative technique described above.

\section{Limitations}

This study is limited by its retrospective nature and by a relatively small patient cohort, despite spanning a 20 -year period at a high-volume surgical practice. As a result, the lack of a direct comparison group is a weakness of the study. Additionally, the length of clinical follow-up varied widely among our study patients, which is a possible source of bias.

\section{Conclusions}

When a patient previously operated on for syringomyelia associated with a CM-I requires reoperation for persisting, recurrent, or expanding syringomyelia, placement of a stent from the fourth ventricle to the cervical subarachnoid space is an effective surgical technique that leads to long-term syrinx decompression in the majority of such patients. This operative technique is well worth considering when the neurosurgeon is confronted with these challenging patients.

\section{Acknowledgments}

We would like to acknowledge The Lucas Warner Fund, The Fellows Family Fund, and The Oxley Research Fund for supporting this project.

\section{References}

1. Cinalli G, Renier D, Sebag G, Sainte-Rose C, Arnaud E, Pierre-Kahn A: Chronic tonsillar herniation in Crouzon's and Apert's syndromes: the role of premature synostosis of the lambdoid suture. J Neurosurg 83:575-582, 1995

2. Dyste GN, Menezes AH, VanGilder JC: Symptomatic Chiari malformations. An analysis of presentation, management, and long-term outcome. J Neurosurg 71:159-168, 1989

3. Sacco D, Scott RM: Reoperation for Chiari malformations. Pediatr Neurosurg 39:171-178, 2003 
4. Strahle J, Muraszko KM, Buchman SR, Kapurch J, Garton HJ, Maher CO: Chiari malformation associated with craniosynostosis. Neurosurg Focus 31(3):E2, 2011

5. Venes JL, Black KL, Latack JT: Preoperative evaluation and surgical management of the Arnold-Chiari II malformation. J Neurosurg 64:363-370, 1986

\section{Disclosures}

The authors report no conflict of interest concerning the materials or methods used in this study or the findings specified in this paper.

\section{Author Contributions}

Conception and design: Scott. Acquisition of data: both authors. Analysis and interpretation of data: both authors. Drafting the article: both authors. Critically revising the article: both authors. Reviewed submitted version of manuscript: both authors.

Approved the final version of the manuscript on behalf of both authors: Scott. Study supervision: Scott.

\section{Correspondence}

R. Michael Scott: Boston Children's Hospital, Boston, MA. michael.scott@childrens.harvard.edu. 